\title{
Acute myocardial infarction with normal coronary artery (MINCA) in a patient with multiple sclerosis
}

\author{
Murat Celik $^{1 *}$, Mehmet Ata Akil ${ }^{2}$, Mustafa Tuncer ${ }^{2}$ \\ ${ }^{1}$ Department of Cardiology, School of Medicine, Gulhane Military Medical Academy, Ankara, Turkey \\ ${ }^{2}$ Department of Cardiology, Van Medicalpark Hospital, Van, Turkey \\ Email: ${ }^{\text {drcelik00@,hotmail.com }}$
}

Received 24 August 2013; revised 25 September 2013; accepted 9 October 2013

Copyright (C) 2013 Murat Celik et al. This is an open access article distributed under the Creative Commons Attribution License, which permits unrestricted use, distribution, and reproduction in any medium, provided the original work is properly cited.

\begin{abstract}
Interestingly, some patients with ST elevation myocardial infarction have normal coronary artery anatomy at cooronary angiography. This situation is called as MINCA (acute myocardial infarction with normal coronary artery). The incidence of MINCA varies between $1.0 \%$ and $8.5 \%$. Although some reasons have been propounded, the exact underlying mechanism remains unclear. Nevertheless, thoracic myelitis can be as a possible cause of myocardial infarction. Herein, we report a case of acute myocardial infarction with normal coronary artery in a patient with multiple sclerosis.
\end{abstract}

Keywords: Acute Myocardial İnfarction; Normal Coronary Artery; Multiple Sclerosis

\section{INTRODUCTION}

Although it is known that rupture of atherosclerotic plaque and subsequent intra-luminal thrombus formation is the main cause of acute myocardial infarction, some patients have normal coronary arteries at coronary angiography in the setting of acute myocardial infarction. The existence of acute myocardial infarction despite angiographically normal coronary arteries (MINCA) was recognized for more than 30 years and its incidence varied between $1.0 \%$ and $8.5 \%$ [1-5]. The aetiology and pathogenesis of the condition are still a source of debate. Some reasons such as cocaine ingestion, coronary vasospasm, endothelial dysfunction, thrombosis, embolization and inflammation, coagulopathies (inherited or acquired), perimyocarditis and stress cardiomyopathy (Takotsubo syndrome) may explain some of these occurrences [3]. Additionally, myocardial infarctions may have been caused by vasospastic reactions secondary to spinal cord

"Corresponding author. pathology such as thoracic myelitis.

Abnormal cardiac function has been described after upper-spinal-cord lesions due to acute autonomic imbalance by the disruption of sympathetic pathways [6]. Nevertheless, coronary vasospastic reactions secondary to spinal cord pathology may lead to myocardial infarction [7-9]. However, the number of case report descriging the relationship between myocardial ischemia and multiple sclerosis (MS) is limited and pathogenesis of this condition is still being debated. We hereby wanted to draw attention to this rare togetherness by reporting a case of MINCA in a patient with MS and tried to explain the relationship between MINCA and MS.

\section{CASE REPORT}

A 40-year-old woman without a history of any cardiovascular disease was admitted to our hospital because of typical chest pain for 1 hour. She had a history of MS for 5 years. An admission 12-lead electrocardiogram (ECG) showed ST elevation in leads II, III, aVF and reciprocal changes in leads V1,V2, suggesting an acute inferior myocardial infarction (Figure 1). Vital parameters and standart biochemical tests at admission were with in normal ranges. Coronary angiography (CAG) was performed. Interestingly, CAG did not reveal any stenosis, luminal irregularities, thrombus or coronary spasm in any of 3 coronary arteries (Figures 2(a) and (b)). Additionally, we performed aortography for the suspicion of occlusion of any anomalous coronary artery, but there was not a coronary artery anomaly. The patient took in coronary intensive care unit and treated with infusion of unfractioned heparin and glyceryl trinitrate at recommended dose for the possibility of coronary vasopasm and/or spontenous resolution of thrombus and embolization into the microvascular integrity. Cardiac enzymes were elevated 2.5 hours later: peak creatine kinase 3045 IU/L; peak creatine kinase MB $405 \mathrm{IU} / \mathrm{L}$; and troponin, 


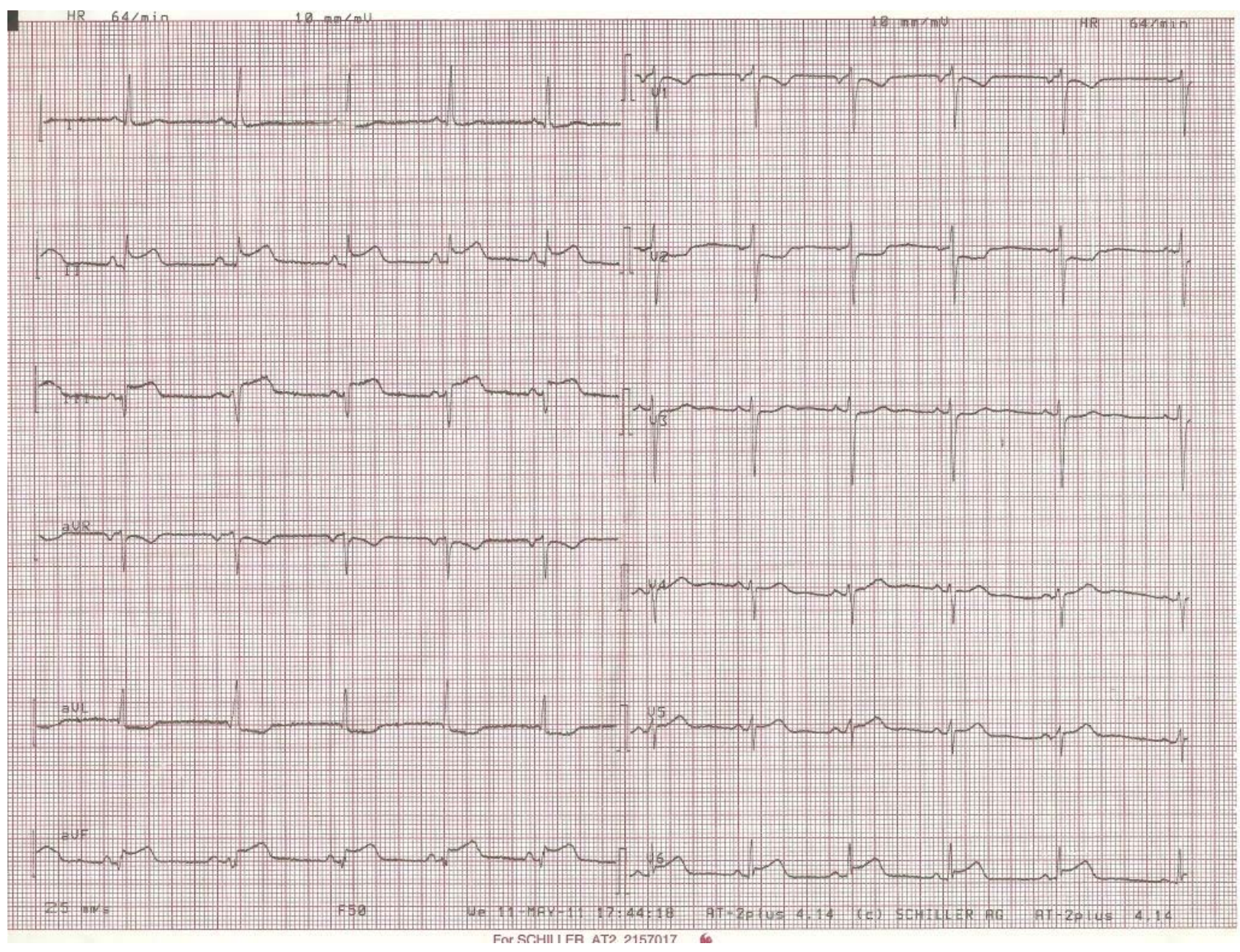

Figure 1. 12-lead electrocardiogram (ECG) at admission shows ST elevation in leads DII, DIII and aVF.

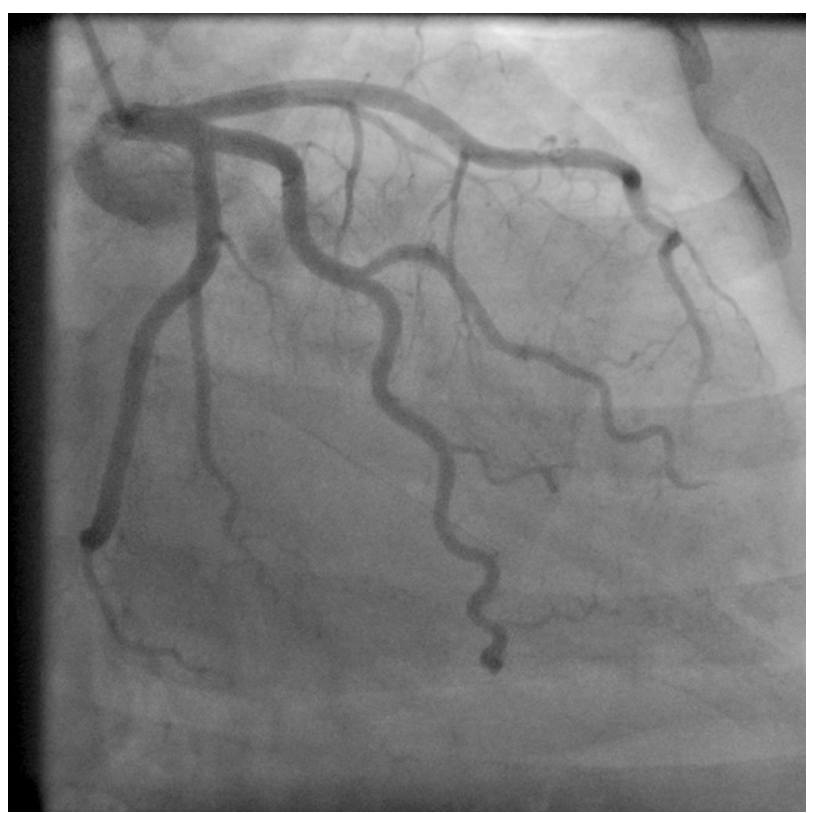

(a)

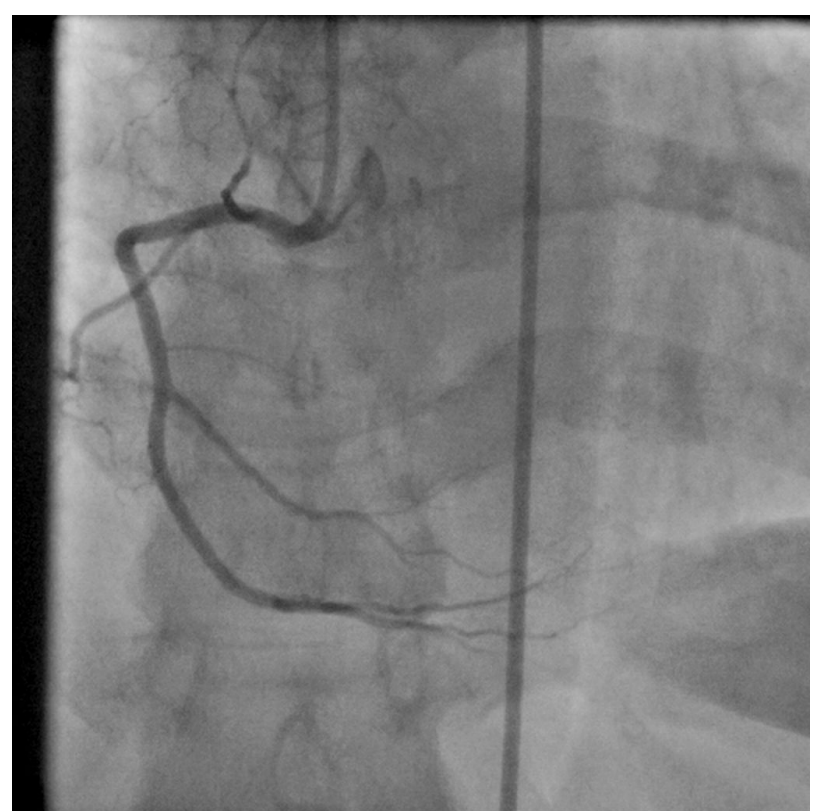

(b)

Figure 2. Left (a) and right (b) coronary angiograms show normal coronary artery. 
$7.22 \mathrm{ng} / \mathrm{mL}$. The erythrocyte sedimentation rate was 16 $\mathrm{mm} / \mathrm{h}$, total leukocyte count was 9600 cells $/ \mu \mathrm{L}, \mathrm{C}$-reactive protein level was $0.3 \mathrm{mg} / \mathrm{dl}$. Echocardiogram showed inferior hypokinesis without any finding of pericarditis. Her chest pain recurred and same ECG finding suggesting an acute inferior myocardial infarction were found at the 2 nd day of hospitalization. CAG repeated and revealed normal coronary arteries once more. At that time, she also complained of dizziness, nausea and vomiting. Magnetic resonance imaging (MRI) was performed and revealed multiple foci of increased T2 signal intensity, but there was not any finding of new foci suggesting MS exacerbation. The patient was treated with infusion of methylprednisolone at recommended dose for five days. Her all complaints including chest pain did not occurred again during hospitalization. On the 4th day of hospitalization, creatine kinase was $164 \mathrm{IU} / \mathrm{L}$; creatine kinase $\mathrm{MB}$ was $32 \mathrm{IU} / \mathrm{L}$; and troponin was $2.63 \mathrm{ng} / \mathrm{mL}$. Then, the patient was discharged in a very good condition on the 7 th day of hospitalization.

We presumed that the patient had an acute myocardial infarction. We thought about the possibility of coronary vasospasm or intra-luminal thrombosis resolved spontaneously. However, either clear atherosclerosis or thrombus formation was not present at CAG. Thus, we could not point out the underlying mechanism exactly.

\section{DISCUSSION}

MINCA is a rare but fair clinical entity. Patients with MINCA are younger and less likely to have traditional risk factors for atherosclerosis than those of identifiable CAD [3]. The incidence and the underlying etiology of MINCA remain unclear. A number of possible mechanisms have been suggested. The most known are coronary vasospasm and thromboembolic disease. Changes in autonomic neural control, changes in contractile function, locally released vasoactive mediators in contact with dysfunctional endothelium, history of smoking, mental stress and oral contraceptive use may be responsible for coronary artery vasospasm [7]. In their study, Bulkley et al. [10] described the autopsy findings of nine patients with progressive systemic sclerosis and evidence of ischemic heart disease but morphologically normal coronary arteries. They observed contraction band necrosis, as a form of reperfusion lesion, in seven of nine patients, and suggested that intermittent vascular spasm at some level of the microcirculation would account for the contraction band type of necrosis and cause myocardial infarction with morphologically normal coronary arteries in patients with progressive systemic sclerosis. Larsen et al. [3] showed a trend toward a higher prevalence of ma lignancy in patients with MINCA, and suggested that malignancy - induced hypercoagulability or inflammation - might be the underlying mechanism of MINCA. Also, myocarditis is one of the possible causes of MINCA, especially in patients below the age of 35 years [11]. Other potential mechanisms are cocaine ingestion, coagulopathies (inherited or acquired), stress cardiomyopathy or Takotsubo syndrome, myocardial bridging and spontaneous coronary artery dissection $[1,12,13]$.

Sympathetic innervation of the heart originates from the cervical and the upper four or five thoracic segments of the spinal cord and has an important role in regulating cardiac function and myocardial blood flow [14]. Abnormalities of cardiac function have been described after acute injuries to the cervical spinal cord and/or spinal cord pathologies such as thoracic myelitis. Lehmann et al. [6] evaluated cardiac functions after acute injury to the spinal cord in 71 consecutive patients and they found that acute severe injury to the cervical spinal cord is regularly accompanied by arrhythmias (persistent bradycardia, marked sinus slowing, supraventricular arrhythmias) and hemodynamic abnormalities (hypotension) resolved spontaneously within 2 to 6 weeks. They suggested the primary mechanism underlying these observations appears to involve the acute autonomic imbalance created by the disruption of sympathetic pathways located in the cervical cord [6].

Multiple sclerosis (MS), also known as disseminated sclerosis or encephalomyelitis disseminate, is an inflammatory autoimmune disorder in which the body's own immune system destroys the fatty myelin sheath protects and insulates the neurons of the brain and the spinal cord. MS is typically a relapsing-remitting neurologic disorder and usually occurs in young adults, and it is more common in women [15]. Nevertheless, patients with MS have been shown to be at risk for various forms of cardiovascular dysfunction related to involvement of reflex pathways in the brainstem and parasympathetic dysfunction [16]. Additionally, coronary vasospasm and/or myocardial ischemia can be seen in patients with MS and normal coronary arteries. A search of the literature reveals only 3 case reports of indicating the relationship between myocardial infarction and spinal cord pathology. Lalouschek et al. [8] reported a 53-year-old woman with normal coronary arteries who had angina at rest, which was associated with myelitis of the thoracic spinal cord. Joing et al. [7] reported a case of 38-year-old woman who experienced Prinzmetal variant angina during a multiple sclerosis relapse. Lappegard et al. [9] reported myocardial infarction in a 45-year-old male with acute and documented inflammatory changes in the spinal cord. In all of the 3 case reports, the thoracic spinal cord lesion led to coronary vasoconstriction due to the fact that intense activation of cardiac sympathetic nerves had been suggested as the most plausible explanation by authors. In our patients, the coronary arteries were angiographically completely normal indicating the fact that patients' 
symptoms might be due to vasospastic coronary occlusion and no obvious cause could be found except MS. We did not observe any significant neurological finding related to patient's lower or upper extremities. So, we did not perform MRI of spinal cord. Although we did not perform a MRI of spinal cord, a lesion in the stated segments of the spinal cord might be the cause of the findings of myocardial ischemia in our patient.

Patients with MINCA have a significantly better prognosis than those of angiographically documented coronary artery disease (CAD) [3]. Long-term outcomes appear favourable in the majority of patients [1]. It was reported that there was no recurrence of anginal symptoms in any of the MINCA patients and no patient underwent a revascularization procedure within 1 year $[1$, 3]. However, patients' chest patin was repeated 2 days later in our case indicating acute myocardial infarctions in the same coronary territories. The optimal treatment of MINCA is currently controversial. There is no consensus about the use of heparin, aspirin, antiplatelet drugs and statins, even after the angiographic finding of normal coronary arteries. It is reasonable to make a cause-spesific treatment. It was shown that no further episodes of angina pectoris were observed after administration of high dose methylprednisolone treatment in patient with MS [8].

In conclusion, MINCA is a rare entity, but its incidence seems to be more increased than expected by the frequent use of CAG. The prognosis of this condition is much better than myocardial infarction with coronary artery disease, and prognosis is likely variable according to the underlying mechanism. Cardiologist should be aware of the possible underlying mechanism in the setting of the MINCA, because treatment is likely variable according to the underlying mechanism. Multiple sclerosis may be one of these possible explanations.

\section{REFERENCES}

[1] Ahmar, W. and Lefkovits, J. (2008) Acute ST elevation myocardial infarction with angiographically normal coronary arteries: Causes and outcomes. International Journal of Cardiology, 128, 131-133.

http://dx.doi.org/10.1016/j.ijcard.2007.05.053

[2] Ammann, P., Marschall, S., Kraus, M., et al. (2000) Characteristics and prognosis of myocardial infarction in patients with normal coronary arteries. Chest, 117, 333338. http://dx.doi.org/10.1378/chest.117.2.333

[3] Larsen, A.I., Galbraith, P.D., Ghali, W.A., et al. (2005) Characteristics and outcomes of patients with acute myocardial infarction and angiographically normal coronary arteries. American Journal of Cardiology, 95, 261-263. http://dx.doi.org/10.1016/j.amjcard.2004.09.014

[4] Sharifi, M., Frohlich, T.G. and Silverman, I.M. (1995) Myocardial infarction with angiographically normal coronary arteries. Chest, 107, 36-40.

\section{http://dx.doi.org/10.1378/chest.107.1.36}

[5] Gehani, A.A., Al-Mulla, A.W., Chaikhouni, A., et al. (2001) Myocardial infarction with normal coronary angiography compared with severe coronary artery disease without myocardial infarction: The crucial role of smoking. Journal of Cardiovascular Risk, 8, 1-8. http://dx.doi.org/10.1097/00043798-200102000-00001

[6] Lehmann, K.G., Lane, J.G., Piepmeier, J.M., et al. (1987) Cardiovascular abnormalities accompanying acute spinal cord injury in humans: Incidence, time course and severity. Journal of the American College of Cardiology, 10, 46-52. http://dx.doi.org/10.1016/S0735-1097(87)80158-4

[7] Joing, S., Casey, R. and Forgosh, L. (2004) Prinzmetal variant angina associated with multiple sclerosis. Journal of the American Board of Family Medicine, 17, 71-73. http://dx.doi.org/10.3122/jabfm.17.1.71

[8] Lalouschek, W., Muller, C., Gamper, G., et al. (1997) Myocardial ischemia with normal coronary arteries associated with thoracic myelitis. The New England Journal of Medicine, 337, 1920.

http://dx.doi.org/10.1056/NEJM199712253372612

[9] Lappegard, K.T., Justad, G., Prytz, J.F., et al. (2005) Thoracic myelitis as a possible cause of myocardial infarction. Journal of Internal Medicine, 257, 558-560. http://dx.doi.org/10.1111/j.1365-2796.2005.01487.x

[10] Bulkley, B.H., Klacsmann, P.G. and Hutchins, G.M. (1978) Angina pectoris, myocardial infarction and sudden cardiac death with normal coronary arteries: A clinicopathologic study of 9 patients with progressive systemic sclerosis. American Heart Journal, 95, 563-569. http://dx.doi.org/10.1016/0002-8703(78)90297-1

[11] Agewall, S., Eurenius, L., Hofman-Bang, C., et al. Myocardial infarction with angiographically normal coronary arteries. Atherosclerosis, 95, 398-400.

[12] Kardasz, I. and De Caterina, R. (2007) Myocardial infarction with normal coronary arteries: A conundrum with multiple aetiologies and variable prognosis: An update. Journal of Internal Medicine, 261, 330-348. http://dx.doi.org/10.1111/j.1365-2796.2007.01788.x

[13] Wittstein, I.S., Thiemann, D.R., Lima, J.A., et al. (2005) Neurohumoral features of myocardial stunning due to sudden emotional stress. The New England Journal of Medicine, 352, 539-548. http://dx.doi.org/10.1056/NEJMoa043046

[14] Di Carli, M.F., Tobes, M.C., Mangner, T., et al. (1997) Effects of cardiac sympathetic innervation on coronary blood flow. The New England Journal of Medicine, 336, 1208-1215. http://dx.doi.org/10.1056/NEJM199704243361703

[15] Noseworthy, J.H., Lucchinetti, C., Rodriguez, M., et al. (2000) Multiple sclerosis. The New England Journal of Medicine, 343, 938-952. http://dx.doi.org/10.1056/NEJM200009283431307

[16] Acevedo, A.R., Nava, C., Arriada, N., et al. (2000) Cardiovascular dysfunction in multiple sclerosis. Acta Neurologica Scandinavica, 101, 85-88.

http://dx.doi.org/10.1034/j.1600-0404.2000.101002085.x 